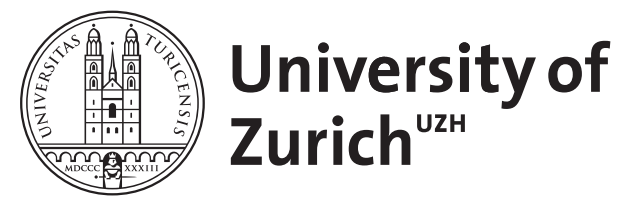

\title{
La philologie romane en Suisse
}

Trachsler, Richard

\begin{abstract}
In the academic landscape of Switzerland, the existence and place of Romance philology may, at first glance, appear traditional, that is, conforming to its German origins. In reality, however, there is a Swiss approach to Romance philology, which appeared with the introduction of the discipline to the University of Zurich in 1872. The present article provides a retrospective of the arrival and activities of Gustav Gröber, the first to hold the Chair of Romance Philology in Zurich, as well as the emergence of a Swiss model, distinct from its German mould. Included in the annex is a selection of documents from the archives of the University of Zurich relating to the Chair of Gröber.
\end{abstract}

DOI: https://doi.org/10.1515/zrp-2016-0071

Posted at the Zurich Open Repository and Archive, University of Zurich ZORA URL: https://doi.org/10.5167/uzh-171926

Journal Article

Published Version

Originally published at:

Trachsler, Richard (2016). La philologie romane en Suisse. Zeitschrift für romanische Philologie, 132(4):938957.

DOI: https://doi.org/10.1515/zrp-2016-0071 


\title{
Richard Trachsler \\ La philologie romane en Suisse
}

\section{Du cas particulier à l'exception}

\begin{abstract}
In the academic landscape of Switzerland, the existence and place of Romance philology may, at first glance, appear traditional, that is, conforming to its German origins. In reality, however, there is a Swiss approach to Romance philology, which appeared with the introduction of the discipline to the University of Zurich in 1872. The present article provides a retrospective of the arrival and activities of Gustav Gröber, the first to hold the Chair of Romance Philology in Zurich, as well as the emergence of a Swiss model, distinct from its German mould. Included in the annex is a selection of documents from the archives of the University of Zurich relating to the Chair of Gröber.
\end{abstract}

Keywords: Romance philology, Gustav Gröber, history of universities, Romance philology in Switzerland

Mots-clés : philologie romane, Gustav Gröber, histoire des universités, philologie romane en Suisse

\section{Introduction}

Comme d'autres pays européens comme l'Italie et l'Espagne, la Suisse abrite sur son territoire trois langues romanes différentes. Le français, l'italien et le rhétoroman sont, en Suisse, des langues nationales qui sont aujourd'hui implantées à tous les niveaux administratifs et politiques, avec une visibilité quotidienne sans faille qui va du billet de banque au panneau de rue. Toutefois, à quelques exceptions près, en général dues à un opportunisme politique circonstanciel, elles ne sont pas perçues comme liées par leur origine commune, mais simplement comme minoritaires par rapport à une langue dominante qui est l'allemand. Chacune de ces langues romanes se définit d'abord par rapport à sa grande cousine germanique et ensuite seulement, à la rigueur, par rapport à ses sœurs romanes.

Adresse de correspondance: Prof. Dr. Richard Trachsler, Universität Zürich, Romanisches Seminar, Zürichbergstr. 8, CH-8032 Zürich, E-Mail: richard.trachsler@uzh.ch 
L'enseignement universitaire en Suisse offre, bien entendu, un reflet exactement inverse : quand naît la philologie romane en tant que discipline universitaire, elle naît à Zurich en territoire germanophone, et l'intitulé même, Romanische Philologie, démontre que les trois langues sont mises sur un même plan, ensemble avec l'espagnol, le portugais et quelques autres, justement parce qu'elles sont toutes sœurs. C'est que, on le sait, la philologie romane n'est pas une invention de Zurich, mais de Bonn. ${ }^{1}$ Or, quand on regarde depuis l'Allemagne dans une perspective humboldtienne, on voit d'abord la famille et ensuite les individus, tout comme la compréhension des membres individuels de cette famille passe par la compréhension de l'évolution parcourue depuis leur origine latine commune jusqu'à l'état présent. La chaire créée en 1872 à Zurich et occupée par l'Allemand Gustav Gröber est en effet le reflet fidèle de celle créée en 1867 à Berlin et occupée par le Suisse Adolf Tobler. ${ }^{2}$

Gröber ne resta à Zurich que quelques semestres, aussi mécontent de son salaire et de ses conditions de travail que les étudiants zurichois étaient mécontents du français oral du maître. La philologie romane « à l'allemande », elle, est restée, instaurant un paradigme scientifique par rapport auquel, nolens volens, les universités en territoire roman ont dû se positionner et qui, toujours nolens volens, s'est modifié depuis 1872.

Un retour dans les années 1860 permet de se faire une idée très précise des défis et des difficultés qui attendent en 2015 un cursus de philologie romane en Suisse, puisque les spécificités helvétiques, en particulier en Suisse alémanique, sont restées inchangées pendant cent cinquante ans et continuent à influencer fortement les contours de la discipline. Les langues romanes, en Suisse alémanique, ne sont pas des langues étrangères. Même si presque personne ne les parle et que quasiment tout le monde, y compris les instances qui décident des volumes horaires consacrés à l'enseignement des langues dans le secondaire, donne au quotidien et dans la « vraie vie » la priorité à l'anglais, elles restent dans notre inconscient - de manière diffuse, mystérieuse, mais tenace - des langues nationales. Elles font partie de la Suisse et sont indispensables à l'idée que nous nous faisons de notre nation.

1 Hirdt (1993).

2 Sur Tobler et la chaire de Berlin, voir Lebsanft (2009) et Kalkhoff (2010, 143-147). 


\section{Gustav Gröber et les origines de la Philologie Romane à l'université de Zurich}

L'université de Zurich a été fondée en 1833 dans l'essor économique et républicain caractéristique d'une certaine Suisse, entrepreneuriale et bourgeoise. ${ }^{3}$ On connaît la célèbre « définition » de ce qu'est une discipline universitaire $:$ il y a discipline universitaire quand il y a un professeur ordinaire pour l'enseigner. Dans ce sens, il ne pouvait y avoir de philologie romane à Zurich avant son institutionnalisation à Berlin en 1867 à travers la chaire de Tobler. Néanmoins, avant même l'invention de la philologie romane comme discipline universitaire, on proposait, à Zurich, des cours touchant certains pans de la philologie romane au sens très large. C'était surtout le fait des Privatdozenten, à qui l'université accordait, et accorde encore, après une procédure d'habilitation, une très faible rétribution et la venia legendi, qui s'éteint si la personne ainsi habilitée n'en fait pas usage. Ces Privatdozenten, sans être très nombreux, proposaient un enseignement dont « l'offre [...] excédait assurément la demande $»{ }^{4}$ Pour l'italien, on compte Francesco Gidoni (1790-1862), de Venise, chargé de cours en droit, Ludwig Herkules Daverio (1804-1849), de Milan, professeur de langue à la Industrieschule à Zurich et journaliste pour la Neue Zürcher Zeitung. Pour le français intervenaient des personnages comme Jacques-Antoine Sangrain de Beaumajin (1777- ?), ${ }^{5}$ ancien précepteur du prince Maximilien de Leuchtenberg, ou François-Armand Saintes (1801- ?), pasteur de l'Église française de Zurich. Mais en l'absence d'étudiants à proprement parler, il n'existait pas de public. Si, par exemple, le Cours de lecture française de Sangrain eut lieu au semestre d'été 1834, aucun de ceux qu'il annonça jusqu'au semestre d'hiver 1841, soit sur une durée de six ans, ne put se

3 Le point de départ pour toute recherche sur l'université de Zurich est l'excellent volume jubilaire Gagliardi/Nabholz/Strohl (1938, 718-724), où sont traitées la période et la matière qui nous concernent. Un tour d'horizon très complet se lit chez Wartburg (1913). On consultera également avec profit la grande somme de Fryba-Reber (2013, 105-122). La liste complète officielle des enseignements proposés à l’Université de Zurich est accessible en ligne : <http://www.histvv. uzh.ch/> [consulté le 7 décembre 2015].

4 C'est l'élégante formule de Fryba-Reber $(2013,112)$.

5 C'est le patronyme complet proposé par Fryba-Reber (2013, 112), le Vorlesungsverzeichnis de l'université de Zurich, indique, moins noblement : « Sangrain, Jakob Anton. ^1777 ; von Beaunay, Frankreich. S 1833-S 1835 PD für franzSpr und Lit. » (Gagliardi/Nabholz/Strohl 1938, 986). Parmi les Privatdozenten plus ou moins éphémères qui possédaient une venia legendi en langue et littérature françaises ou italiennes dans ces premières décennies suivant la fondation de l'université, il faut aussi mentionner : « Granier, Adolf. *1804 ; von Montpellier, Frankreich. Im W 1836 PD für franzSpr und Lit. ». 
tenir. Les proportions sont comparables pour les autres cours proposés à la même époque par ces enseignants. ${ }^{6}$ À côté des Privatdozenten, des titulaires de chaires de disciplines voisines pouvaient occasionnellement proposer un cours sur un sujet en relation avec une langue ou une œuvre littéraire de langue romane. En général, c'étaient des classicistes ou des germanistes qui, accessoirement, pénétraient sur les terres de la future romanistique. Ces « incursions » étaient tout à fait normales puisque la Neuphilologie en tant que discipline universitaire commençait à peine à se détacher de la philologie classique et que les frontières disciplinaires, au sein du trio néophilologique - langues germaniques, romanes et anglaise - étaient donc très poreuses, même si la Germanistik commençait à être dotée, au moins dans les pays germanophones, de chaires spécifiques. Ainsi, le classiciste Johann Kaspar von Orelli (1787-1849) proposait, au semestre d'été 1833, une Spiegazione dell'Orlando furioso di M. Lodovico Ariosto, ou, au semestre d'hiver 1841, un cours sur les Satire di Messer Lodovico Ariosto. Plus tard, le germaniste Ernst Moritz Ludwig Ettmüller (1802-1877), qui avait pour habitude de faire cours déguisé en barde germain, professait, au semestre d'été 1856, un cours sur la Geschichte der epischen Poesie bei den germanischen und romanischen Völkern im Mittelalter. ${ }^{7}$

Ce n'est qu'avec Alfred Rochat (1833-1910) que les cours dans le domaine de la philologie romane prennent une certaine régularité. Après une thèse rédigée sous la direction du germaniste Ettmüller, Rochat obtient une venia legendi à l'ancienne, c'est-à-dire d'orientation néophilologique, pour romanische und germanische Sprachen. ${ }^{8}$ Originaire de Vevey, doté d'un curriculum international et véritablement surprenant, il assura en tant que Privatdozent, de 1857 à 1872, un enseignement assez varié et constant en littérature ancienne et philologie, surtout, mais pas exclusivement, gallo-romane. ${ }^{9}$ En 1872, Rochat, qui était financièrement indépendant, quitta l'université et changea de vie. L'année 1872 vit l'arrivée de Gröber, le début de l'institutionnalisation de la philologie romane, la fin de l'ère des dilettantes. ${ }^{10}$

6 Wartburg (1913, IV 10).

7 Fryba-Reber $(2013,113)$.

8 Rochat fait l'objet d'une remarquable entrée sur Wikipedia, qui permet de compléter les renseignements rassemblés par Gagliardi/Nabholz/Strohl $(1938,719)$.

9 Wartburg (1913, IV 11), mentionne aussi le cas de Johann Jacob Honegger, qui annonça des cours entre 1859 et 1862 qui ne rencontrèrent aucun succès, ce qui l'amena à se retirer de l'Université, puis à demander une seconde fois son habilitation en 1870-1871, pour pouvoir une nouvelle fois proposer des cours, sans rencontrer davantage d'écho.

10 La production de Rochat est d'ailleurs très valable. Il avait étudié avec le germaniste Ettmüller à Zurich et publié plusieurs ouvrages et de nombreux articles. Il est surtout connu pour ses liens 
Avec Gustav Gröber arrive le premier vrai universitaire, issu du système allemand avec un diplôme de romaniste et une habilitation en voie d'achèvement qui devait être la première habilitation à l'université de Zurich dans cette nouvelle discipline. ${ }^{11}$ Gröber a étudié à Leipzig auprès d'Adolf Ebert. ${ }^{12} \mathrm{Il}$ a obtenu son titre de docteur grâce à un travail sur le Fierabras, travail remarquable et remarqué même en France. ${ }^{13}$ Mais il a surtout trouvé grâce aux yeux d'Adolf Tobler, qui lui recommande de déposer une demande d'habilitation à Zurich, au détriment d'Edmund Stengel et parce que Hugo Schuchardt, d'abord pressenti, n'était, paraît-il, pas disponible. ${ }^{14}$ Gröber, issu d'un milieu sinon modeste - son père possède une imprimerie -, en tout cas peu compréhensif à l'égard de ses études, travaille alors comme professeur particulier et plus tard comme enseignant de français à l'école de commerce de Leipzig. Il est déjà fiancé, mais pas encore marié, ${ }^{15}$ et accepte donc, soucieux de ne pas grever le budget parental, un poste à l'université de Zurich pour le début du semestre d'été 1871, malgré le salaire modique de 1500 francs, d'abord en tant que Privatdozent, ensuite en tant que professeur extraordinaire.

Il tient sa leçon inaugurale le 29 avril 1871, quelques jours avant son vingtseptième anniversaire, et commence alors la carrière que l'on sait. Gustav Gröber est resté cinq semestres à Zurich, avant d'accepter une chaire à l'université de Breslau, de 1874 à 1879, puis à Strasbourg, où il allait rester trois décennies. ${ }^{16}$

d'amitié avec l'écrivain Conrad Ferdinand Meyer. On trouvera la liste de ses livres dans la bibliographie infra, Rochat $(1855,1856,1859,1874)$.

11 Adolf Ebert, Doktorvater de Gröber, et Adolf Tobler, qui joua le rôle d'éminence grise dans beaucoup de recrutements à Zurich jusqu'à sa mort, recommandaient l'acceptation de ce travail. Cf. Curtius $(1951,260)$.

12 Historien de par sa formation, Adolf Ebert fait partie de ces savants, issus des disciplines voisines, appelés à occuper les toutes premières chaires de philologie romane. Il était l'éditeur du Jahrbuch für romanische und englische Literatur, ce qui lui a valu d'être appelé sur la nouvelle chaire de philologie romane à Leipzig en 1862. Sur Ebert, voir Bochmann (1997).

13 Gröber (1869). Pour une bibliographie de la réception voir Curtius (1951, 260), Formisano (1979, en particulier les pages 250-254) et Formisano (2013).

14 Voir les deux documents édités infra en annexe.

15 C'est ainsi que j'interprète le passage chez Curtius (1951, 260), qui utilise le terme de Braut mais parle plus loin de "Junggesellenmahlzeiten» (ib.), et, à la fin (ib., 261), de « eigener Hausstand », en l'an 1875.

16 Sur le plan personnel, l'intégration de Gröber en Suisse n'a pas été cauchemardesque. Curtius (1951, 260) rappelle qu'il s'était pris de passion pour l'escalade et qu'il s'était fait quelques connaissances, entre autres Gottfried Keller. Cela est confirmé par l'écrivain zurichois, qui écrit à son ami Adolf Exner, professeur de droit romain, le 20 décembre 1873 pour le tenir au courant des affaires zurichoises : « Gröber ist nach Breslau berufen und von Regierungsrat Sieber eigenhändig in der Zeitung belobt worden, dass er mit Dank und Anerkennung von Zürich scheide (nicht wie 
Pour bien comprendre le caractère éphémère du passage de Gröber à Zurich, il faut se rappeler que les conditions matérielles que pouvaient offrir les jeunes universités suisses étaient nettement inférieures à celles qui étaient pratiquées en Allemagne ou en Autriche. Cela explique non seulement la brièveté du séjour de Gröber aux bords de la Limmat, mais aussi celle de Hermann Suchier, qui le remplaça, et qui ne resta que le seul semestre d'hiver 1874-1875, avant d'aller à Münster, puis Halle. Le successeur de Suchier, Franz Gustav Settegast, resta à Zurich de 1878 à 1883, généralement mécontent, au point de refuser un renouvellement de son poste aux mêmes conditions. ${ }^{17}$ Pour cette génération d'Allemands, Zurich était un lieu de transit. ${ }^{18}$ Le premier titulaire de philologie romane à rester plus longtemps fut le Suisse Heinrich Morf. Après douze ans passés à Zurich (1889-1901), il quitta quand même la ville pour Francfort, puis Berlin. ${ }^{19}$

Voici donc la liste des enseignements de Gröber à Zurich, « von denen keine einzige unbesucht blieb ${ }^{20}$

\begin{tabular}{ll}
\hline Semestre & Vorlesungen \\
\hline SS 1871 & Erklärung von Corneille's Horace \\
\hline SS 1871 & Altfranzösische Grammatik mit Übungen \\
\hline WS 1871 & Italienische Grammatik \\
\hline WS 1871 & Erklärung und kritische Behandlung altfranzösischer Texte \\
\hline WS 1871 & Syntaxe française \\
\hline SS 1872 & Dante, Inferno \\
\hline SS 1872 & $\begin{array}{l}\text { Provenzalische Grammatik mit Leseübungen nach Bartsch, Chrestomathie } \\
\text { provençale, }{ }^{\mathrm{e}} \text { Edit., Elberfeld 1868 }\end{array}$ \\
\hline SS 1872 & Romanische Gesellschaft \\
\hline WS 1872 & $\begin{array}{l}\text { Erklärung provençalischer Denkmäler nach Bartsch, Chrestomathie } \\
\text { provençale, Elberfeld 1868 }\end{array}$ \\
\hline WS 1872 & Spanische und portugiesische Grammatik \\
\hline
\end{tabular}

Boretius und Bennford). Er hatte nämlich ein sentimentales Entlassungsgesuch geschrieben im obigen Sinne » (Keller 1927, 30).

17 Gagliardi/Nabholz/Strohl (1938, 721 n. 1). Son salaire était de 3000 francs, soit le double de ce qu'avait touché le jeune Gröber.

18 Fryba-Reber $(2013,114)$ parle d'une « chaire-tremplin ».

19 Sur Morf, je me permets de renvoyer à mon étude, Trachsler (2009).

20 Wartburg (1913, IV 11). La formule, je crois, veut dire que tous les cours ont eu lieu et non qu'il y a eu foule. 


\begin{tabular}{ll}
\hline Semestre & Vorlesungen \\
\hline SS 1873 & Vergleichende Grammatik der romanischen Sprachen \\
\hline SS 1873 & $\begin{array}{l}\text { Altfranzösische Grammatik mit Leseübungen (nach Bartsch, Chrestomathie } \\
\text { de l'ancien français, Leipzig 1872) }\end{array}$ \\
\hline SS 1873 & Romanische Gesellschaft \\
\hline WS 1873 & Geschichte der französischen Literatur im Mittelalter \\
\hline WS 1873 & Erklärung des Romancero del Cid, nebst Einleitung \\
\hline WS 1873 & Romanische Gesellschaft \\
\hline
\end{tabular}

Cette palette d'enseignements est tout à fait conforme à ce qui se proposait à la même époque en Allemagne. ${ }^{21}$ Rien de moderne ou de contemporain, un peu de littérature de l'Âge Classique, beaucoup d'ancien français et d'occitan ainsi que du Moyen Âge en général, puis régulièrement de l'italien avec un peu d'espagnol et de portugais en alternance. On a indubitablement affaire à une offre en philologie romane. La nouveauté est qu'elle est proposée par un seul homme, un romaniste, par opposition d'une part aux Privatdozenten de la génération précédente, dont la venia legendi englobait une seule langue, et, d'autre part, par opposition aux anciens Sprachmeister, qui assuraient autrefois, dans l'université ou dans les Ritterakademien allemandes, l'enseignement des langues modernes, au même titre que les maîtres de danse ou d'équitation, d'ailleurs bien mieux rémunérés que les professeurs de langue. Ces Sprachmeister étaient en général des natifs speakers à la biographie aventureuse et ne disposaient pas de diplôme universitaire, ce qui leur valait le regard condescendant des professeurs universitaires. Leur enseignement se limitait à la pratique, négligeant la dimension théorique ou historique. ${ }^{22}$ La pierre angulaire du nouveau système est donc bien le nouveau professeur, le romaniste qui forme des étudiants selon les règles de l'art et les nouvelles techniques, qui, comme les lois phonétiques ou le stemma codicum, mettent un terme à des siècles de dilettantisme.

21 Presque toutes les universités d'Allemagne ont mis en ligne leur Vorlesungsverzeichniss historique. L'offre est assez homogène du point de vue de la qualité et varie surtout en fonction des moyens à disposition.

22 Voir, pour l'exemple de Göttingen, Krapoth (2001). Certains éléments sont repris, en français, dans Krapoth (2013). Pour un contexte plus large, voir L'enseignement du français en Europe autour du XIX $X^{e}$ siècle. Histoire professionnelle et sociale, numéro thématique de Documents pour l'histoire du français langue étrangère ou seconde 33/34 (2005), <http://dhfles.revues.org/1664> [consulté le 15 juin 2013]. 


\section{Du modèle allemand vers la spécificité suisse}

Ce modèle allemand va, mutatis mutandis, assez rapidement devenir le modèle suisse : après l'université de Zurich en 1872, Bâle suit en 1877, Berne en 1879, Lausanne en 1888, encore dans le cadre de l'Académie, la fondation de l'université datant de 1891, puis Fribourg en 1889, avec une chaire de langues et littératures romanes et une seconde, pour la langue et littérature françaises, qu'occupera Bédier. Genève nomme en 1891 Ernest Muret sur une chaire de langues et littératures romanes, mais bien avant cette date, Eugène Ritter s'occupait de l'histoire de la langue française. L'Académie de Neuchâtel est la dernière à créer un enseignement de philologie romane, en 1895, mais propose dès 1873 un enseignement de grammaire historique du français. ${ }^{23}$

En schématisant un peu, on dira donc qu'en Suisse alémanique, les créations des chaires de philologie romane et des institutions en général sont un peu plus précoces qu'en Romandie et privilégient très nettement la dimension romane, alors qu'en Suisse romande, ces chaires sont parfois précédées ou flanquées d'un enseignement institutionnalisé du français.

Restons encore un instant à Zurich, à l'époque de Gröber, avant d'examiner comment la discipline a évolué depuis ce moment fondateur. Tout allemand qu'il est, le système zurichois a en effet une spécificité qui s'est faite sentir dès le départ et dont Gröber a un peu pâti. Dès avant sa nomination, le français parlé de Gröber avait soulevé des doutes auprès des autorités et, plus tard, auprès des étudiants. ${ }^{24}$ En Allemagne, ce n'était pas un problème et le grand Diez lui-même aurait, diton, déclaré «Ich spreche kein Französisch ${ }^{25}$ En Suisse, c'était différent, il s'agissait d'une langue nationale et il fallait atteindre un certain standard. D'ailleurs, le titulaire de la chaire avait aussi la responsabilité de la formation des futurs enseignants des lycées et, surtout, des Sekundarschulen. Les jeunes gens qui se destinaient à l'enseignement dans ces derniers établissements n'avaient aucun besoin de connaître la phonétique historique et l'étymologie, mais devaient correctement maîtriser une langue parce qu'ils devaient l'enseigner à leur tour à des élèves qui allaient l'écrire ou la parler, ordinairement, dans un cadre commercial. En général, à cette époque, c'était le français moderne, dont il fallait une connaissance ni théorique ni historique, mais pratique. Dans le cas de Gröber, le point avait été soulevé dès la lettre de recommandation de Tobler, au

23 Voir, à ce propos, Fryba-Reber $(2009,51)$.

24 Gagliardi/Nabholz/Strohl (1938, 719, n. 2).

25 Friedrich Diez, cité d'après Hirdt (1993, 8). 
moment de décrire les qualités et les limites des candidats à son collègue Schweizer-Sidler, qui organisait la procédure de recrutement côté zurichois :

« Herr Gröber kennt übrigens die Lücken seines Wissens genau und würde namentlich auch es für seine Pflicht halten, an der Förderung seiner Tüchtigkeit im praktischen Gebrauche des Französischen weiter zu arbeiten, was ihm ja in der Schweiz nicht schwer fallen würde $» .^{26}$

À l'attention des autorités politiques, Schweizer-Sidler, linguiste hautement respecté à son époque, résume la situation avec toute la clarté souhaitable :

« Ich meinte, dass die Überzeugung bei den hohen Räthen durchgedrungen sei, dass kaum zwei Fliegen - hier einer, der gut französisch parliert und ein Philologe auf dem Gebiete der romanischen Sprache - mit einem Schlage gefangen werden können.

Ist es zumeist [?] für die Lehramtscandidaten nöthig, ausser Rambert noch einen Lehrer der ersteren Art zu gewinnen dann, meinte ich, würde das der hiesige französ. Pfarrer oder ein anderer geborener Wälscher oder Franzosen versehen. Einen Franzosen oder Wälschen, der die romanischen Sprachen wissenschaftlich durchforscht, bekommen wir nicht ; ob ein Deutscher, der beide Vorzüge vereinigt, zu erhalten ist, muss ich sehr bezweifeln. Der Unterrichtsart [ ?] als solcher steht aber natürlich nur ein Mann an, der in unserem Sinne, der wissenschaftlich verfährt. Was nun Herrn Dr. Groeber speziell betrifft, so kann ich die über ihn gestellten Fragen nicht bestimmt beantworten, so viel meinte ich aber aus Freund Toblers Brief leicht herauszulesen, dass die praktische mündliche Handhabung der romanischen Sprachen nicht seine Stärke sei, dass er sich aber $\left[\mathrm{v}^{\circ}\right]$ darin durch Ferienaufenthalte in den der Schweiz benachbarten romanischen Ländern etc. [ ?] vervollkommen werde. Ich hoffe, dass wir, wenn wir die wissenschaftliche Pflege der romanischen Sprache bei uns

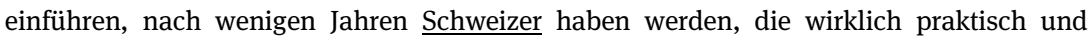
wissenschaftlich zugleich in diesem Felde allen billigen Anforderungen entsprechen ${ }^{27}{ }^{27}$

Il n'existe pas, sur ce marché des années 1870, le mouton à cinq pattes qu'on cherche : on aura soit un Français ou un Romand a-scientifique, soit un Allemand savant, incapable de bien parler la langue. C'est l'un ou l'autre. Mais la synthèse est attendue pour bientôt : les Suisses - alémaniques - « die wirklich praktisch und wissenschaftlich zugleich in diesem Felde allen billigen Anforderungen entsprechen ». C'est cette idée même qui guide encore aujourd'hui nos recrutements et qui explique que nos moutons à cinq pattes n'ont jamais cinq pattes.

Certes, ce clivage entre universitaires et praticiens du secondaire existe aussi en Allemagne, mais, sauf erreur, jamais ces Schulmänner n'ont reproché à un universitaire allemand de ne pas maîtriser une langue, même dans les débats parfois houleux qui opposaient les universitaires qui prônaient une approche

26 Universitätsarchiv der Universität Zürich, U 109.6.48 olim U 109 d1.46. Pour la transcription complète et quelques notes d'éclaircissement, voir infra, l'édition en annexe.

$27 \mathrm{Ib}$. 
holistique de romaniste, fondée sur des questionnements théoriques et historiques, et les représentants des Schulmänner allemands qui réclamaient des programmes simples et orientés vers la pratique d'une seule langue. C'est qu'aucune de ces langues romanes n'était la leur et que, quelque part, ils n'aspiraient pas à les apprendre au point de devenir des natifs speakers. ${ }^{28}$

$\mathrm{Au}$ contraire, comme le montre l'anecdote suivante. En 1879, quelques années après le départ de Gröber de Zurich, on cherche, à Strasbourg, un successeur pour Eduard Boehmer. Un député alsacien émet alors l'idée que le prochain titulaire devrait non seulement maîtriser la langue française oralement et à l'écrit, mais devrait aussi être capable de donner ses cours en français. La réaction des universitaires allemands ne se fait pas attendre :

« Die französischen und sonstigen ausländischen romanischen Philologen, die irgend diesen Namen verdienen, sind, weil man ohne Kenntniss des Deutschen romanische Philologie überhaupt nicht studiren kann, sämmtlich der deutschen Sprache mächtig, und für wen soll denn da der deutsche Romanist in einer andern als seiner Muttersprache schreiben, wenn er sicher ist, von allen Fachgenossen in dieser verstanden, ja sogar besser verstanden zu werden ? $"{ }^{29}$

Le poste de Strasbourg, on le sait, est allé à Gröber, qui, après un passage à Breslau, où il avait fondé la Zeitschrift für romanische Philologie, y est resté trente ans. À Strasbourg, il a lancé le Grundriss et formé des élèves comme Ernst Robert Curtius ou Philip August Becker. Il n'est donc pas indispensable de savoir s'exprimer un peu correctement en français pour être un excellent romaniste.

L'on voit donc dès le début une certaine spécificité de la romanistique suisse par rapport à sa grande sœur allemande. Encore aujourd'hui, les cours sont donnés dans la langue dont relève la matière : langue et littérature françaises en français, langue et littérature italiennes en italien, langue et littérature espagnoles en espagnol, etc. ${ }^{30}$ On peut qualifier cette façon de faire de " tradition ", car il n’y a sans doute aucun texte légal qui empêcherait de proposer un cours de

28 Voir les très intéressantes pages chez Kalkhoff (2010, 271-274) sur les efforts de certaines universités de proposer une praxisnahe formation et les déboires de Morf à la tête de la toute nouvelle Akademie für Sozial- und Handelswissenschaften, qui défend un enseignement plus moderne, prévoyant des séjours à l'étranger et la familarisation avec la culture contemporaine, à qui son ami Tobler écrit sèchement depuis Berlin qu'il ne voit aucune raison d'envoyer des étudiants de Berlin à Francfort.

29 Eduard Koschwitz, Elsaß-Lothringische Zeitung du 22 avril 1880, n 94 . L'épisode est célèbre, il a été commenté dans l'introduction de l'œuvre de Hirdt (1993, 3), d'après lequel nous citons.

30 La même chose est vraie, de façon spéculaire, de la Suisse romande, où les cours de langue et littérature allemandes sont donnés en allemand, etc. Cette pratique ne se limite toutefois pas aux langues nationales, mais concerne aussi, par exemple, l'anglais. 
littérature française en allemand dans une université germanophone, tout comme on ne peut pas empêcher, à ma connaissance, un étudiant qui souhaiterait passer son examen en allemand de le faire. Mais cette tradition est aujourd'hui très généralement respectée et remonte, sans doute, aux origines de l'université, au moins pour ce qui concerne les exercices pratiques. On a vu que Gröber annonçait, en français, Syntaxe française, et il devait probablement exister une forme de code non écrit en faveur de l'enseignement dans la langue de la matière puisque Heinrich Morf se plaint, dans une lettre adressée à Gaston Paris après avoir quitté Berne pour Zurich, de devoir traduire ses cours de l'allemand en français. ${ }^{31}$ De la même époque, c'est-à-dire de 1889 , date un extrait du protocole de la faculté faisant état des conclusions de la commission responsable de pourvoir la chaire qui allait échoir à Morf. Le document prouve que, jusque-là, la coutume était bien d'enseigner dans la langue de la matière :

« Die Commission wünscht ferner, daß in Zukunft die Vorlesungen wenigstens in der Regel in deutscher Sprache gehalten werden, namentl[ich] deshalb, weil der Gewinn für die Studierenden größer werden muß, wenn der Docent seine ganze Kraft der Sache zuwenden kann ; in den praktischen Kursen sollen dagegen die fremdsprachlichen Übungen energisch betrieben werden ${ }^{32}$

Il y a donc eu comme une " modernisation ", une mise à jour de la vieille pratique où la romanistique zurichoise s'est très temporairement alignée sur l'Allemagne. Si la nouvelle recommandation a pu concerner Morf, déjà son successeur, le Vaudois Ernest Bovet, revint naturellement au français pour l'ensemble de son enseignement portant sur la littérature française et se réservant l'allemand pour la littérature italienne..$^{33}$ Derrière la question de la langue de l'enseignement qui peut paraître un détail purement formel et superficiel se cache en réalité un élément-clé de la philologie romane suisse par rapport à celle d'Allemagne : ce ne sont pas, pour nous, des langues étrangères, ce sont les nôtres et il faut que le titulaire de la chaire les domine non pas passivement ou théoriquement, mais dans la pratique.

De là découle probablement aussi la seconde spécificité que permet de faire apparaître l'évolution des chaires zurichoises après Gröber. À côté de celle qu'occupaient les savants allemands éphémères - Gröber, Suchier, Settegast -, on en avait créé une seconde en 1876, pourvue par Johann Jakob Breitinger, surtout

31 Voir infra, notre annexe, pour l'édition du passage.

32 Zürich, Staatsarchiv, Z 70.2897 [Protokoll der Philosophischen Fakultät I, 1860-1894], 182s., séance du samedi 18 mai 1889.

33 Fryba-Reber $(2013,122)$. 
pour les besoins de la formation des futurs enseignants du secondaire. ${ }^{34}$ Sa chaire avait un profil néophilologique à l'ancienne avec anglais, italien, français et espagnol, et il enseignait la littérature, mais aussi la langue. D’après les témoignages, Breitinger maîtrisait admirablement toutes ses langues, mais il est évident qu'avec cette charge d'enseignement, sa production scientifique était forcément moins imposante. Lorsqu'il décède, en 1889, seul professeur ordinaire pour la Neuere Philologie, la faculté décide de moderniser le profil et de séparer l'enseignement de l'anglais de celui des langues romanes, préludant de quelques années à la création physique d'un Englisches et d'un Romanisches Seminar qui jusque-là étaient regroupés dans un même institut. ${ }^{35}$

« Die Professur soll nicht mehr wie bisher Französisch, Italienisch und Englisch umfaßen, sondern es soll in Anbetracht der Unmöglichkeit, daß bei dem heutigen Stande der Wissenschaft ein Mann die drei Sprachen und Literaturen in gleicher Weise beherrsche, das Englische abgetrennt werden ${ }^{3}{ }^{36}$

On a donc mis au concours la chaire de philologie romane qu'allait occuper Morf. En outre, la discipline était représentée par Jacob Ulrich, qui avait hérité de l'extraordinariat de philologie romane que Franz Settegast avait libéré en 1883, lorsqu'il avait quitté l'université de Zurich dépité par les conditions financières médiocres. En dédiant de très bonne heure deux chaires à la philologie romane, l'université de Zurich ouvrait le champ à la spécialisation de la discipline. C'est ici le second point où la philologie romane en Suisse paraît se distinguer à la fois de la France et de l'Allemagne et trouver une réelle singularité.

\section{L'émergence d'un modèle suisse}

Depuis les temps de Gröber s’est opérée une première spécialisation évidente, qui a naturellement concerné l'ensemble des universités occidentales : c'est la scission de la discipline qui s'appelait jadis Romanische Philologie en Romanische Sprachwissenschaft d'un côté, qu'on désigne, en français, par « linguistique romane », et de l'autre en Romanische Literaturwissenschaft, qu'on glose parfois par « littératures romanes », comme s'il n’y avait pas de terme pour désigner le fait de s'occuper scientifiquement de littérature. À Zurich, c'est chose faite dès le

34 Gagliardi/Nabholz/Strohl (1938, 720s.); Fryba-Reber (2013, 118s.).

35 Voir à ce propos le volume commémoratif du centenaire du Romanisches Seminar, Wüest (1997).

36 Zürich, Staatsarchiv, Z 70.2897 [Protokoll der Philosophischen Fakultät I, 1860-1894], 182s., séance du samedi 18 mai 1889. 
départ de Morf : on a d'un côté le « littéraire » Ernest Bovet et en face le « linguistique » Louis Gauchat, même si la dénomination des chaires n'a pas été adaptée. ${ }^{37}$ Mais ce sera officiel, et transcrit dans l'intitulé des chaires, à partir de la génération suivante, c'est-à-dire depuis les années 1930, où l'on a les littéraires Theophil Spoerri et Reto R. Bezzola et les linguistes Jakob Jud et Arnald Steiger. ${ }^{38}$ Ce n'est donc pas là que se situe la spécificité helvétique. Tous les départements de philologie romane comptent aujourd'hui des linguistes et des littéraires. La spécificité helvétique réside plutôt dans le haut degré de spécialisation au sein des différentes philologies nationales : à côté de différentes chaires de linguistique romane avec, de facto, une forte délimitation par langue, où les collègues évitent d'empiéter sur le terrain du voisin, nous avons des chaires de littérature italienne, littérature ibérique, littérature rhéto-romane, littérature française et même une chaire pour la littérature française et occitane du Moyen Âge. En d'autres termes, nous avons une spécialisation tellement poussée que la Romanistik telle qu'elle est comprise et conçue en Allemagne se trouve compartimentée en autant de Einzelphilologien. Mais il s'agit toujours, au moins pour le rhétoroman, l'italien et le français, des nôtres.

Elles correspondent aussi - et le fait a son importance - aux langues qui sont enseignées dans les lycées. La correspondance est presque parfaitement symétrique, alors que le modèle allemand soulève un problème souligné par Hans Rheinfelder dans un célèbre article de 1960 :

« Die deutschen Universitäten haben Lehrstühle für Deutsche Philologie ; an den höheren Schulen Deutschlands gibt es Deutsch-Unterricht. Die Universitäten haben Lehrstühle für Englische Philologie ; an den höheren Schulen gibt es Englisch-Unterricht. Die Universitäten haben Lehrstühle für Romanische Philologie, d.h. für die Geschichte der neun romanischen Sprachen und Litteraturen ; an den höheren Schulen gibt es Unterricht im Französischen, Spanischen und Italienischen » (Rheinfelder 1960, 201).

Cette « efficacité » des Einzelphilologien qui reflètent exactement les langues qui sont enseignées dans les lycées explique partiellement le succès de cette formule. La complexité de la matière " philologie romane ", difficile à compacter, fait le reste. Comment couvrir sérieusement la linguistique et la littérature de trois, quatre, cinq ou six langues si le titulaire de la chaire n'a que six ou huit heures d'enseignement à sa disposition?

37 Gagliardi/Nabholz/Strohl $(1938,854)$.

38 Spoerri a succédé à Bovet en 1922 et Jud à Gauchat en 1926. Steiger est devenu Privatdozent en 1925, professeur extraordinaire en 1933, tandis que Bezzola est devenu Privatdozent en 1929, puis professeur extraordinaire en 1938. Les deux ont été promus au rang de professeur ordinaire après la guerre, en 1945. 
Pour ces raisons, il est rare de trouver en Suisse un enseignement de la philologie romane, entendons de la vraie philologie romane, celle qui faisait écrire à Gröber cette phrase pleine d'arrogance et de dédain, à propos de l'université de Budapest, où l'on cherchait en 1893 un professeur de français : "Wahrscheinlich kennt man in Pest eine romanische Philologie nicht $"{ }^{39}$ Pouvons-nous aujourd'hui arguer de la présence de l'inscription Romanisches Seminar sur la porte de nos instituts et nos cartes de visite pour considérer que nous sommes des romanistes et partager encore la fierté de Gröber d'être si supérieurs au simple gallo-romaniste, ibéro-romaniste ou italo-romaniste ?

À Zurich, il existe un cursus de Langues romanes comparées et un autre en Littératures romanes comparées. Le premier a été créé au moment de la réforme de Bologne, en 2006, au niveau du Bachelor, puis élargi au Master en 2008. C'est aussi le moment où l'on a créé le Master en Littératures romanes comparées. Les deux cursus sont composés, au sens étymologique du terme, de modules qui appartiennent au cursus du français, de l'italien, de l'espagnol, du portugais, du roumain, etc. En d'autres termes, à l'exception d'une liste de lecture ad hoc, il n'existe pas d'enseignement spécifiquement conçu pour ces cursus. Ils ne nous coûtent donc rien. C'est l'aspect positif. Est-ce qu'ils marchent ? Sauf erreur, on a eu, depuis leur création, dix-huit étudiants en Bachelor et cinq en Master pour le cursus de Langues romanes comparées et zéro en Littératures romanes comparées, jusqu'en 2015, où s'est inscrite une doctorante ... venue d'Allemagne. ${ }^{40}$

Il est clair que ce n'est plus la philologie romane que nous avons apprise. Celle qui éclaire, celle qui explique, celle qui seule permet de comprendre chacune de ses composantes. La discipline qui remplissait d'orgueil Gustav Gröber n'est pas la juxtaposition de modules empruntés à d'autres filières, mais un contenu et une forme qui se complètent. Nous avons poussé la spécialisation trop loin et essayons maintenant en vain de recoller les morceaux sans y mettre l'argent nécessaire. La philologie romane vaut mieux que ce que nous lui offrons à Zurich et on peut se réjouir de voir que c'est la Suisse romande qui s'efforce de lui insuffler aujourd'hui une nouvelle vie. Je souhaite de tout cœur à la nouvelle filière lausannoise le succès qu'elle mérite.

39 Lettre de Gröber à Becker du 25 mai 1893, citée d'après Hillen (1993, 155). Il faut savoir, naturellement, que Gröber essaie de placer son élève Becker à l'université de Budapest. Avec succès, d'ailleurs.

40 Je remercie mes collègues Annina Clerici et Reto Zöllner d'avoir bien voulu vérifier pour moi les chiffres et les dates. 


\section{Bibliographie}

Bochmann, Klaus, Sprachwissenschaftler wider Willen: Adolf Ebert und die Anfänge der Leipziger Romanistik, in: Haßler, Gerda/Storost, Jürgen (edd.), Kontinuität und Innovation. Studien zur Geschichte der romanischen Sprachforschung vom 17. bis zum 19. Jahrhundert. Festschrift für Werner Bahner zum 70. Geburtstag, Münster, Nodus, 1997, 275-289.

Curtius, Ernst Robert, Gustav Gröber und die romanische Philologie, Zeitschrift für romanische Philologie 67 (1951), 257-288 [reimprimé dans id., Gesammelte Aufsätze zur romanischen Philologie, Bern/München, Francke, 1960, 428-455].

Formisano, Luciano, Alle origini del lachmannismo romanzo. Gustav Gröber e la redazione occitanica del «Fierabras », Annali della Scuola Normale Superiore di Pisa. Classe di Lettere e Filosofia, ser. III, 9:1 (1979), 247-302.

Formisano, Luciano, La romanistique allemande et l'Italie au XIX siècle, in: Trachsler, Richard (ed.), Bartsch, Foerster et $C^{i e}$. La première romanistique allemande et son influence en Europe, Paris, Classiques Garnier, 2013, 129-143.

Fryba-Reber, Anne-Marguerite, De Gustav Gröber à Arthur Piaget (1872-1895). L'institutionnalisation de la philologie romane en Suisse, in: Bähler, Ursula/Trachsler, Richard (edd.), Portraits de médiévistes suisses (1850-2000). Une profession au fil du temps, Genève, Droz, 2009, 33-58.

Fryba-Reber, Anne-Marguerite, Philologie et linguistique romanes : institutionnalisation des disciplines dans les universités suisses (1872-1945), Leuven, Peeters, 2013.

Gagliardi, Ernst/Nabholz, Hans/Strohl, Jean (edd.), Die Universität Zürich 1833-1933 und ihre Vorläufer. Festschrift zur Jahrhundertfeier, Zürich, Verlag der Erziehungsdirektion, 1938.

Gröber, Gustav, Die handschriftlichen Gestaltungen der Chanson de geste «Fierabras » und ihre Vorstufen, Leipzig, Vogel, 1869.

Hillen, Ursula, Wegbereiter der Romanischen Philologie: Ph. A. Becker im Gespräch mit G. Gröber, J. Bédier und E. R. Curtius, Frankfurt am Main, Lang, 1993.

Hirdt, Willi, Romanistik. Eine Bonner Erfindung, 2 vol., Bonn, Bouvier, 1993.

Hugo Schuchardt Archiv, <http://schuchardt.uni-graz.at/> .

Kalkhoff, Alexander, Romanische Philologie im 19. und frühen 20. Jahrhundert. Institutionengeschichtliche Perspektiven, Tübingen, Narr, 2010.

Keller, Gottfried, Aus Gottfried Kellers glücklicher Zeit. Der Dichter im Briefwechsel mit Marie und Adolf Exner, Wien, Speidl, 1927.

Krapoth, Hermann, Die Beschäftigung mit romanischen Sprachen und Literaturen an der Universität Göttingen im 18. und frühen 19. Jahrhundert, in: Lauer, Reinhard (ed.), Philologie in Göttingen. Sprach- und Literaturwissenschaft an der Georgia Augusta im 18. und beginnenden 19. Jahrhundert, Göttingen, Vandenhoeck \& Ruprecht, 2001, 57-90.

Krapoth, Hermann, Les premiers séminaires de philologie romane. L'exemple de Göttingen, in: Trachsler, Richard (ed.), Bartsch, Foerster et $C^{i e}$. La première romanistique allemande et son influence en Europe, Paris, Classiques Garnier, 2013, 23-36.

Lebsanft, Franz, Adolf Tobler (1835-1910). I : " Der gesamte Reichtum der Menschennatur », in: Bähler, Ursula/Trachsler, Richard (edd.), Portraits de médiévistes suisses (1850-2000). Une profession au fil du temps, Genève, Droz, 2009, 61-95.

Rheinfelder, Hans, Vom Notstand der romanischen Philologie, Die Neueren Sprachen, Neue Folge 9 (1960), 201-208.

Rochat, Alfred, Über einen bisher unbekannten Percheval li Galois. Eine literarhistorische Abhandlung, Diss. Zürich, Druck von E. Kiesling, 1855. 
Rochat, Alfred, Drei Schweizerdichter aus dem dreizehnten Jahrhundert, Heidelberg, Rieger, 1856.

Rochat, Alfred, Bertran de Born. Étude sur un poète du douzième siècle, Vevey, Eymann, 1859. Rochat, Alfred, Ein altladinisches Gedicht in Oberengadiner Mundart, Zürich, Schabelitz, 1874.

Rudolf, Winfried, Bernhard ten Brink and Edmund Max Stengel - two pupils of Friedrich Diez and their contributions to the study of medieval English in the national discourse of the nineteenth century, in: Trachsler, Richard (ed.), Bartsch, Foerster et $C^{i e}$. La première romanistique allemande et son influence en Europe, Paris, Classiques Garnier, 2013, 223-246.

Trachsler, Richard, Heinrich Morf (1854-1921). Le bâtisseur déchu, in: Bähler, Ursula/Trachsler, Richard (edd.), Portraits de médiévistes suisses (1850-2000). Une profession au fil du temps, Genève, Droz, 2009, 141-75.

Wartburg, Walther von, Schweiz. Zürich, Kritischer Jahresbericht über die Fortschritte der romanischen Philologie 12 (1913), IV 9-31.

Wüest, Jakob (ed.), Les Linguistes suisses et la variation linguistique, Basel/Tübingen, Francke, 1997.

\section{Annexe ${ }^{41}$}

\section{" Einer, der gut französisch parliert ». L'importance du français à l'Université de Zurich}

Nous présentons ici une partie du dossier concernant le recrutement de Gröber à Zurich, d'après les pièces originales conservées dans les archives universitaires de Zurich. Il s'agit des tout premiers pas entrepris par la faculté. C'est une lettre d'Adolf Tobler adressée à Schweizer-Sidler, le président de commission de recrutement, et une seconde lettre que ce dernier écrit à un responsable de l'Instruction Publique, qui résume la situation d'un point de vue non scientifique. L'intérêt des deux pièces est d'évoquer ouvertement le problème de l'enseignement des langues romanes à l'université de Zurich, qui se voit confrontée à la difficulté de devoir choisir entre les compétences scientifiques et les connaissances pratiques des candidats.

Pour la bonne compréhension des lettres, il est utile de connaître le contexte: d'après ce qui se laisse reconstituer, les Zurichois ont contacté Tobler, professeur à Berlin, mais qui avait étudié avec Schweizer-Sidler à Zurich, et dont le frère occupait la chaire de linguistique germanique. Visiblement, le premier plan était d'approcher le jeune Hugo Schuchardt, à Leipzig. Tobler, de passage à Leipzig, n'a pas pu voir Schuchardt, absent, mais prend langue avec Ebert, le romaniste

41 Je remercie Marco Veneziale pour son aide dans la préparation de cette annexe. 
de Leipzig, qui lui recommande son élève Gröber, dont Tobler connaît et apprécie la thèse.

La lettre de Tobler, ainsi que celle de Schweizer-Sidler, mentionne le nom de Stengel. Il s'agit du futur professeur de Marbourg, un des romanistes les plus actifs de tous les temps. C'est que Stengel, lui aussi à la recherche d'un poste après avoir soutenu sa thèse, envisageait de se faire habiliter à Zurich, dans l'espoir de la création d'un poste. Tobler et Schweizer-Sidler, qui ont déjà leur préférence, le dissuadent. Le moins qu'on puisse dire c'est que les trois noms qui surgissent dans ce dossier - Schuchardt, Stengel, Gröber - ont tous joué un rôle de premier plan dans l'histoire de la discipline. ${ }^{42}$

Le troisième extrait ici publié est tiré d'une lettre que Morf adresse à Gaston Paris. Il tire le bilan de sa première année d'activité à l'université de Zurich après avoir quitté Berne, avec l'aide, entre autres, de Gaston Paris. Il évoque, parmi d'autres points, le fait d'avoir à donner ses cours en français.

\section{Le choix de Zurich en faveur de Gröber en 1870}

\section{Lettre de Tobler à Schweizer-Siedler du 2 octobre 1870}

Universitätsarchiv der Universität Zürich, U 109.6.48 olim U 109 d1.46

Verehrter Herr Professor,

Nach Abrede mit Dr. Schuchardt mich über die in Zürich zu besetzende Lehrstelle zu besprechen ist mir leider nicht möglich geworden, da derselbe augenblicklich von Leipzig abwesend ist, welches ich schon morgen verlassen muß. Dagegen habe ich Ebert gesprochen und von ihm erfahren, daß Schuchardt schwerlich Leipzig verlassen werde, wo er nun erst ein Semester und zwar mit erfreulichem Erfolge thätig gewesen ist.

Von jüngeren Leuten empfahl er mir besonders seinen mehrjährigen Schüler und Famulus Dr. Gröber, den nämlichen von dessen trefflicher Dissertation, einer Kritik der verschiedenen Versionen des Fierabras, ich Ihnen viel Rühmliches habe sagen können. Da Dr. Gröber sich gerade in Leipzig aufhält, wo er eine durch den Krieg vorübergehend vacant gewordene Lehrerstelle an der Handelsschule interimistisch ausfüllt, so ließ ich ihn bitten, mich aufzusuchen, und habe heute in längerer Unterredung ihm auseinander gesetzt, was man ungefähr von dem Lehrer für das romanische Fach in Zürich erwarten würde, und in dem nicht mehr ganz jungen Manne $\left[1 v^{\circ}\right]$ einen recht liebenswürdigen, bescheidenen, vielseitig und doch sehr gründlich unterrichteten Fachgenossen kennen gelernt, von dessen Arbeit ich mir wesentliche Förderung der romanischen Studien verspreche und der mir durch die ruhige Sicherheit seines Wesens sich auch zum akademischen Lehrer zu eignen scheint.

42 Sur Schuchardt, voir le très riche Hugo Schuchardt Archiv ; pour Stengel, voir Rudolf (2013, 238-246). 
Er sprach mir seine Geneigtheit aus, den Versuch in Zürich zu wagen und wird auf meinen Rath sich in directe Verbindung mit Ihnen setzen. Ob er sich entschließen wird, nach Zürich über zusiedeln, ohne daß ihm irgend ein Gehalt zugesichert wird, weiß ich nicht ; es schien mir, als gestatteten ihm seine Verhältnisse nicht, auf jedes Honorar zu verzichten ; aber er wird sich gern mit bescheidenem Entgelt begnügen und ist bereit, eine Vorlesung mit besonderer Rücksicht auf die Lehrbedürfnisse der Sekundarlehramtscandidaten zu halten. Vor Ostern jedoch würde er seine Thätigkeit nicht eröffnen - Ueber Dr. Stengel konnte ich Weiteres nicht erfahren ; ich möchte nur das bemerken, daß Gröbers erste Arbeit, die bezeichnete Dissertation, sich dann doch bei weitem höher über das Niveau des Durchschnittes solcher Schriften erhebt, eine ganz andere Selbständigkeit des Verfahrens bekundet, als das wenn auch fleissige, doch mehr nach der Schablone $\left[2 r^{\circ}\right]$ angefertigte Bruchstück einer churwälschen Lautlehre. ${ }^{43} \mathrm{Hr}$. Gröber kennt übrigens die Lücken seines Wissens genau und würde namentlich auch es für seine Pflicht halten, an der Förderung seiner Tüchtigkeit im praktischen Gebrauche des Französischen weiter zu arbeiten, was ihm ja in der Schweiz nicht schwer fallen würde.

Er hat, wie Sie sehen, den vortheilhaftesten Eindruck auf mich gemacht, und ich glaube, die Facultät müßte sich Glück wünschen [?], die ihn gewinnen und festhalten könnte.

Mein Schwiegervater ${ }^{44}$ bittet mich, Sie bestens von ihm zu grüßen.

In treuer Ergebenheit

Ihr Adolf Tobler

Leipzig 2. Okt. 1870

\section{Lettre de Schweizer-Sidler, adressée probablement à Johann Kaspar Sieber, Erziehungsdirektor, peu après avoir reçu la lettre de Tobler en octobre 1870.} Universitätsarchiv der Universität Zürich, U 109.6.48 olim U 109 d1.46

\section{Hochzuverehrender Herr,}

Ich kann es nicht bergen, dass mich Ihre freundlichen Zeilen etwas überrascht haben.

Ich meinte, dass die Überzeugung bei den hohen Räthen durchgedrungen sei, dass kaum zwei Fliegen - hier einer, der gut französisch parliert und ein Philologe auf dem Gebiete der romanischen Sprache - mit einem Schlage gefangen werden können.

Ist es zumeist [?] für die Lehramtscandidaten nöthig, ausser Rambert noch einen Lehrer der ersteren Art zu gewinnen dann, meinte ich, würde das der hiesige französ. Pfarrer oder ein anderer geborener Wälscher oder Franzosen versehen. ${ }^{45}$ Einen Franzosen oder Wälschen, der die romanischen Sprachen wissenschaftlich durchforscht, bekommen wir nicht ; ob ein Deutscher, der beide Vorzüge vereinigt, zu erhalten ist, muss ich sehr bezweifeln. Der Unterrichtsart [?] als

43 Tobler commet ici un lapsus intéressant puisque c'est Schuchardt, et non Stengel, qui venait de présenter, en 1870, sa thèse d'habilitation à Leipzig, « Über einige Fälle bedingten Lautwandels im Churwälschen ».

44 Tobler s'était marié en 1863 avec Ottilie, la fille de l'éditeur Hirzel de Leipzig, avec qui Schweizer-Sidler était en rapport.

45 Eugène Rambert était Professeur ordinaire de littérature française à l'académie de Lausanne (1855-1860), puis à l'EPF de Zurich (1860-1881). Voir la notice du Dictionnaire Historique de la Suisse : <http://www.hls-dhs-dss.ch/textes/f/F16000.php> [consulté le 17 décembre 2015]. 
solcher steht aber natürlich nur ein Mann an, der in unserem Sinne, der wissenschaftlich verfährt. Was nun Herrn Dr. Groeber speziell betrifft, so kann ich die über ihn gestellten Fragen nicht bestimmt beantworten, so viel meinte ich aber aus Freund Toblers Brief leicht herauszulesen, dass die praktische mündliche Handhabung der romanischen Sprachen nicht seine Stärke sei, dass er sich aber $\left[\mathrm{v}^{\circ}\right]$ darin durch Ferienaufenthalte in den der Schweiz benachbarten romanischen Ländern etc. [?] vervollkommen werde. Ich hoffe, dass wir, wenn wir die wissen-

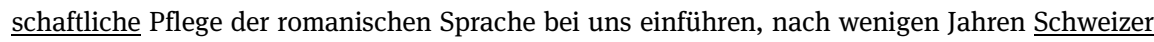
haben werden, die wirklich praktisch und wissenschaftlich zugleich in diesem Felde allen billigen Anforderungen entsprechen.

Ich bitte Sie dringend baldigst eine Antwort - sei es eine zusagende, sei es eine absagende an Dr. Groeber zu veranlassen, welchen übrigens nicht ich, sondern zwei der bedeutendsten Romanisten, Tobler und Ebert, mit voller Überzeugung empfohlen haben, aber allerdings als Mann der Wissenschaft empfohlen haben. Ich ersuche Sie dann auch, mir gef. von der Art der Antwort Notiz zu geben. Fatal, äusserst fatal, ist es [?] freilich, wenn nun weder Groeber noch Stengel kommen. Letzterem hat man zwar noch nicht geantwortet, aber ich habe ihm pflichtmässig geschrieben, dass er wohl in Zürich gefährliche Concurrentz finden möchte und sich vielleicht bewogen fühlte sein Habilitationsgesuch zurückzuziehen.

Zum Schlusse muss ich Ihnen doch sagen, dass ich bis heute noch keine Anzeige von dem letzten Beschlusse des H. Regierungsrathes in meiner Angelegenheit in Händen habe.

Hochachtungsvoll grüsst Sie

Ihr Prof. H. Schweizer

\section{« Diese Seiltänzerei aufstecken ». Morf et les cours en langue française}

\section{Lettre de Morf à Gaston Paris du 21 août 1890 (Extrait)}

Paris, BNF, nouv. acq. fr. 24451

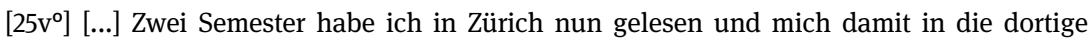
Aufgabe ordentlich eingelebt. Sie ist in manchem von der Berner verschieden und jedenfalls, wenn reicher an Arbeit, so auch angenehmer. Nur der Umstand, dass ich eine Vorlesung über franz. Literatur in französischer Sprache zu halten hatte, war, Bern gegenüber, für mich eine Verschlechterung. Ich glaube zwar, die Sache nicht allzu schlecht gemacht zu haben, aber im Grunde bin ich diesem Theile meiner Aufgabe eben doch nicht gewachsen und dieses Gefühl drückt mich so, dass ich[,] von nun an, diese Seiltänzerei aufstecke. Bloss um Dinge, die ich in meiner Muttersprache ganz ordentlich hätte vortragen können, in schlechtem Französisch einem zu drei Viertheilen deutschen Publikum zu sagen, musste ich mich arg quälen und placken und eine Zeit aufwenden, die ich viel Nützlicher [sic] - z. B. für Gorra $!^{46}$ - hätte ausgeben können. Ich

46 Allusion à un compte rendu du livre d'Egidio Gorra, Testi inediti di Storia Trojana, Torino, Loescher, 1887, que Morf avait promis à Gaston Paris pour la Romania. Le sujet traverse les échanges épistolaires de cette époque jusqu'au moment où, de guerre lasse, Morf finira par 
las über Voltaire (im Winter) ein in der deutschen Redaktion fast druckfertiges Kolleg, das ich nun für Nichts und aber Nichts in's Französische übersetzen musste ; im Sommer traktierte ich eine andere meiner Lieblingsmaterien: die Renaissance des XIX. Jahrhunderts, Staël und Chateaubriand in erster Linie - und die Plackerei war dieselbe. Nun aber habe ich mein Lehrgeld bezahlt ; der Erziehungsrath des Kantons Zürich kann mir jetzt glauben, dass ich für Zürich hinreichend französisch [sic] kann, auch wenn ich meine Vorträge deutsch halte. In den Übungskollegien wird ja nach wie vor immer die fremde Sprache gesprochen.

$[\ldots]$

l'écrire. Il a paru dans la Romania 21 (1892), 88-107, après moult menaces et flatteries de la part de Gaston Paris (pour les flatteries) et de Paul Meyer (pour les menaces). 